\title{
Nasogastric feeding at home: acceptability and safety
}

\author{
C E Holden, J W L Puntis, C P L Charlton, I W Booth
}

\begin{abstract}
A questionnaire was administered to 70 families with experience of home enteral nutrition. All but one patient received at least some of their feeding overnight. During 11041 patient days of home enteral nutrition, no serious complications were seen. Sleep disturbance was common, however, and affected 59 parents and 35 children. A nocturnal cough or 'chestiness' were noted in 10 children suggesting occult gastro-oesophageal reflux. Enteral feeding disposables were not prescribable by general practitioners, and funding for the equipment was inadequate in the hospital and community. The mean time to arrange funding for equipment was eight months (range two weeks to two years). Parental views on home enteral nutrition were positive and none felt that its disadvantages outweighed the benefits. Altogether 35 children were described as being more happy and active. Given adequate preparation and continued support at home, parents can manage home enteral nutrition safely and effectively.
\end{abstract}

Increasingly, we are using enteral nutrition in children at home. This is related in part to an increased awareness of the prevalence of malnutrition among children admitted to hospital with chronic disease. ${ }^{1-5}$ It is also a recognition of the potential benefits of nutritional support in children with chronic disorders such as Crohn's disease, ${ }^{6}$ congenital heart disease, ${ }^{7}$ malignancy, ${ }^{8}$ cystic fibrosis, ${ }^{9}$ renal disease, ${ }^{10}$ and liver disease. ${ }^{11}$ Recent case studies have supported the view that children receiving specialised nutritional support can be nursed effectively at home. ${ }^{12-14}$ Although there is a large body of literature describing the practical techniques, ${ }^{15} 16$ no study has examined the prevalence of complications associated with home enteral nutrition. We therefore undertook to investigate parents' and children's experiences of home enteral nutrition, focusing particularly on any practical difficulties encountered.

The Children's Hospital, Birmingham C E Holden

Institute of Child Health, University of Birmingham J W L Puntis C P L Charlton I W Booth

Correspondence to: Miss C E Holden, The Children's Hospital, Tadywood Middleway, Birmingham B16 8ET. Accepted 26 July 1990 Subjects and methods SUBJECTS

Seventy consecutive families with experience of home enteral nutrition between January 1987 and April 1988 were identified from the records of the nutritional care team at the Children's Hospital. The indications for nutritional support comprised chronic renal failure $(n=8)$, cardiac disease $(n=8)$, cerebral palsy $(n=2)$, malignancy $(n=8)$, chronic liver disease $(n=8)$,
Crohn's disease $(\mathbf{n}=\mathbf{7})$, severe food intolerance $(n=2)$, gastro-oesophageal reflux $(n=10)$, short bowel syndrome $(n=3)$, dysphagia $(n=2)$, and failure to thrive attributable to an inadequate nutritional intake $(n=12)$.

\section{METHODS}

Both parents from each family were questioned by one of us (CEH), either when attending the outpatient clinic or by telephone. The questionnaire was designed to record data about the type of equipment used, and also to explore parents' experiences of managing home enteral nutrition.

\section{PATIENT PREPARATION AND PARENTAL}

\section{TEACHING}

Teaching programmes were tailored individually by the nutritional care sister. In general, however, training always included the safety aspects of enteral feeding, psychological preparation of the child, equipment maintenance, recognition of problems, and guidance on how to cope with them. Interpreters were used as necessary and teaching programmes extended where communication was more difficult. Play therapists helped to prepare the children psychologically for enteral feeding. For toddlers we used play techniques to focus on enteral feeding, and a range of treatment scenarios were prepared. Through play the child was asked about his or her memory of any previous admissions to hospital. We spent a great deal of time with parents, looking not only at the mechanics of enteral feeding but also at the psychological aspects. We gave parents reinforcement literature and toddlers were given a booklet called Roo and Foe, which describes the story of a child receiving nasogastric feeding. The booklet was specifically prepared by staff at the children's hospital and printed with financial support from Sherwood Medical.

Older children were prepared for nasogastric feeding by assessing their level of understanding and perception of their disorder. We explained to them why it was necessary to begin feeding. Time was spent looking at and handling enteral feeding equipment.

A photograph album and video was shown to them, which depicted previous patients passing their own nasogastric tubes. Diagrams in a booklet describing relevant anatomy associated with the digestive and respiratory tracts were discussed with them. They were told about the sensations encountered as the tube is passed and how they could learn to cope with them. They 
were also introduced to other children receiving or who had had enteral feeding.

\section{PATIENT DISCHARGE}

A joint decision was made by medical, nursing, and dietetic staff, and children were discharged when parents were considered competent and confident enough to look after enteral feeding. On discharge from hospital enteral feeding pumps were provided on temporary loan, and one month's supply of disposable equipment was given to parents.

The general practitioner was informed and appropriate district nurses and health visitors were contacted and given details of illness and the reasons for enteral feeding.

The child's parents were given one week's supply of the feed from hospital. The general practitioner was asked to prescribe the feed subsequently.

\section{Results}

The median age (range) at the start of home enteral nutrition was 3.5 years (6 weeks-15 years). A total of 11041 days of home enteral nutrition were studied. Three types of enteral infusion pump were used: Viomedex $(n=34)$, Kangaroo $330(n=34)$, and Cow and Gate $(n=$ 2). Setting up the feeding system at night took a median (range) of five minutes (3-15).

\section{SUPERVISION}

In 59 families, each parent took joint responsibility for setting up the feed and looking after it overnight. In 11 families one parent took sole responsibility, usually the mother. Fifty nine parents got up in the night in order to check on their child and the accuracy of the pump, but in no case did any parent find it necessary to remain awake all night. No nasogastric tube fell out or became displaced into the respiratory tract and disconnection from the giving set was uncommon.

\section{FEEDING REGIMEN}

Forty six of the children received only overnight feeding, and 23 were fed both during day and night. The median (range) duration of feed infusion was 12 hours (4-24). One child with a tracheostomy received feeds only during the day.

\section{NASOGASTRIC TUBES}

Two types of tube was used. Fifty children had polyurethane Silk (Merck) tubes and 20 a polyvinylchloride (PVC) tube (Portex). Silk tubes were used when feeding was expected to last for longer than one month; they were changed monthly and required a guide wire for insertion. PVC tubes were used for short term feeding and were changed weekly as the tube becomes rigid if left longer.

Silk tubes were popular with parents and children because they were soft and easily inserted. However, 10 out of 50 parents using this tube reported that they blocked readily. This was usually prevented by flushing the tube with water after feeds.

Changing the Silk tube only once a month was seen as a clear advantage over PVC tubes. Five children who tended to vomit changed from a Silk to a PVC tube because they found that the more flexible Silk tube was more easily displaced by vomiting. Babies and toddlers often pulled their tubes out when first inserted, but after the first day tolerated them well. Thirty four parents were taught how to pass their child's tube.

The children were taught to pass their own tube at night, removing them in the morning. The median (range) age of these children was 8.75 years (5-15 years). In the remaining families, tubes were passed by hospital nurses, the nutritional care sister, or community nurses.

\section{FAMILY SUPPORT}

In general, parents were satisfied with the level of support offered to them. Parents were told to contact the nutritional care sister and dietetic staff at the hospital with any queries or problems. Sixty families commented that the support provided from the hospital was essential and they welcomed this reassurance. One in six children were visited by a paediatric district nurse who visited parents daily. All parents commented on the excellent care provided. Two thirds of the children under 5 years of age were seen by their health visitor. Six families had received help from a social worker since starting home enteral nutrition. Twenty families reported that community nurses without paediatric training knew very little about nutritional support for children, but in most cases provided helpful moral support. Sixty three families kept in regular telephone contact with members of the nutritional care team. We were able to obtain telephones through social services for families whose children were starting nasogastric feeding. All 20 families whose children were over 2 years of age who required feeding for longer than six months were successful in their application for an attendance allowance.

\section{SUPPLIES}

Disposable equipment for the feeding pumps was given to the parents for the first month at home. In general, there was no recognised budget in the community for disposable equipment and pumps for children at home. The Children's Hospital was therefore required to continue to supply equipment to $\mathbf{4 0}$ of the $\mathbf{7 0}$ families. When it did occur time taken to arrange funding was eight months. Twenty three of the families reported that the sheer weight of feeds caused a problem when carrying them home from the chemist. Eight families initially had problems getting feed from their chemist, as many feeds often required a special order. Dietetic staff often had to contact chemists to organise this. Eight general pratitioners would only give parents a prescription for one week at a time, a practice which they explained on the basis of the cost of the feeds. 
SYMPTOMS RELATED TO HOME ENTERAL NUTRITION

After the introduction of nocturnal nasogastric feeding 35 children suffered from sleep disturbance: related to nocturia $(n=20)$, vomiting $(n=$ $10)$, the need to defaecate $(n=3)$, and abdominal pain $(n=2)$.

Ten children developed a night time cough suggesting possible occult gastro-oesophageal reflux. However, in no cases were symptoms severe enough to warrant stopping feeds. Two children were further investigated and gastrooesophageal reflux confirmed. One of these children was born with oesophageal atresia and tracheo-oesophageal fistula and had had a gastric interposition. She received enteral feeds via a nasojejunal tube, but continued to have reflux and have repeated chest infections; she subsequently had a feeding jejunostomy. The other child presented as an infant with laryngeal stenosis secondary to severe gastro-oesophageal reflux. At the age of 6 years he was started on nasogastric feeding and is awaiting a further antireflux procedure. In neither child was nasogastric feeding thought to have precipitated reflux.

BENEFITS OF HOME ENTERAL NUTRITION

Thirty four parents reported spontaneously that their child appeared more happy and active after the introduction of home enteral nutrition. A similar number spontaneously reported that home enteral nutrition gave them a new sense of freedom. This feeling was mainly the result of not having to devote so much time trying to get their child to eat.

The families with children with complex heart disease felt that home enteral nutrition had prolonged their child's life and enabled them to spend more time together. This comment was echoed by three families of children awaiting liver transplantation. Twenty eight families felt that home enteral nutrition had prevented admission to hospital and allowed care to be given at home.

The nutritional state measured at the start and end of home enteral nutrition demonstrated improvement in all patients in terms of weight for age centile, midarm circumference centile, and arm muscle area.

DISADVANTAGES OF HOME ENTERAL NUTRITION In 36 children the nasogastric tube was the object of unwelcome public interest. Older children were selfconscious and inhibited about going out, and many reported teasing by their peers. This problem was prevented by teaching them to pass their own tube at night and to remove it in the morning.

Two schools were reluctant to allow children with nasogastric tubes to attend, but discussion with the nutritional care team resolved this problem. Thirty nine families reported that initially they were very distressed when their child's tube was passed. However, once in situ the children were not upset by the tube. were no instances of major complications such as aspiration, hypoglycaemia after interruption of feed, or entanglement in the tubing.

\section{Discussion}

Home enteral nutrition is used increasingly in the management of a wide variety of childhood disorders. Although we saw no serious complications, minor problems were common-for example, sleep disturbance affected 60 parents and 35 children. However, overnight feeding enabled the child and their family to lead a more normal daytime lifestyle. ${ }^{17}$

Our policy was to try and make feeding regimens flexible so that family routines were disrupted as little as possible. For example, one mother preferred to restrict feeds to the early evening as her child had a tracheostomy and required frequent tracheal suction overnight, which could provoke vomiting.

Enteral feeding pumps generally functioned well, but further development with regard to portability and alarm systems are still required. ${ }^{18}$ The Viomedex pump has good alarm systems, but parents found it necessary to check the rate of infusion frequently when using this system.

The Kangaroo pump was well accepted by all parents, delivers feed in increments, and has an alarm system which indicates interruption in feed delivery. Unfortunately, the pump is not designed to be portable, but will run from an integral battery for a few hours.

The Cow and Gate pump was used by two patients. This pump is fitted with an alarm and is relatively portable, but unlike the other pumps, there is no carry pack available. Parents reported difficulty finding babysitters willing to cope with a feed pump. We now encourage other relatives and close friends to come to the hospital to be taught feeding techniques so that they can relieve parents from time to time. Silk tubes are routinely used for long term feeding and are well accepted, although the PVC tubes were less likely to become displaced by vomiting.

Ensuring adequate supplies of disposable equipment was often a problem. Such equipment is not available on prescription, and the nutritional care team is not funded to provide equipment for families in the community. Referring hospitals often ignored requests to supply equipment to parents.

It is now the policy of our hospital to write to the unit general managers in the community asking them to provide appropriate equipment. Unfortunately this request is sometimes refused on the grounds of lack of funding.

Parents had to order equipment well in advance from the community agencies and many continued to ask for hospital supplies because of irregular supplies sent. One family obtained disposable supplies directly from the chemist by special arrangement with the health authority.

Guidelines relating to funding and supply of equipment and disposables are urgently needed. ${ }^{19}$ It is clearly an anomaly that general practitioners may prescribe feed products but not the equipment necessary for their 
administration..$^{20}$ In the meantime our findings confirm that home enteral nutrition can be managed both safely and effectively by parents, provided they are given adequate training and support.

We would like to thank all parents and children who contributed to this study.

1 Merritt RJ, Suskind RM. Nutritional survey of hospitalised pediatric patients. Am f Clin Nutr 1979;32:1320-5.

2 Parsons HG, Francoeur TE, Howland P, Spengler RF, Pencharz PB. The nutritional status of hospitalised children. Am $\mathcal{F}$ Clin Nutr 1980;33:1140-6.

3 Cooper A, Jakobowski D, Floyd T, Ziegler MM, Koop E. Nutritional assessment: an integral part of preoperative surgical evaluation. 7 Pediatr Surg 1981;16:554-61.

4 LeLeioko NS, Luder E, Fridman M, Fersel J, Benkov K. Nutritional assessment of pediatric patients admitted to an acute-care pediatric service utilising anthropometric measurements. Foumal of Parenteral and Enteral Nutrition 1986;10:166-8.

5 Moy RJD, Smallman S, Booth IW. Malnutrition in a UK children's hospital. Foumal of Human Nutrition and Dietetics 1990;3:93-100.

6 Aiges H, Markowitz J, Rosa J, Daum F. Home nocturnal supplemental nasogastric feeding in growth-retarded adolescents with Crohn's disease. Gastroenterology 1989;97: 905-10.
7 Bougle SPA, Start KM, Rees L. Nutritional treatment of congenital heart disease. Arch Dis Child 1986;61:799-801. Donaldson SS, Wesley MN, Dewys WD, Suskin RM, Jaffe N, Van Eys J. A study of the nutritional status of pediatric N, Van Eys J. A study of the nutritional status of pediatric

9 MacDonald A, Littlewood JM. Nutritional management of cystic fibrosis. Bromley, Kent: Cystic Fibrosis Research Trust, 1989.

10 Strife CF, Quinlan M, Mearsk K, Davey ML, Clardy C. Improved growth of three uraemic children by nocturnal nasogastric feeding. Am $\mathcal{F}$ Dis Child 1986;140:438-43.

11 Stuart S, Kaufman MD, Nancy D, et al. Nutritional support for the infant with extrahepatic biliary atresia. $\mathcal{F}$ Pediat 1987;110:679-86.

12 Buchanan E, Holden C, Charlton C. Tube feeding an infant. Clinical Nutritional Update 1988;1:7.

13 Garrington C. Fight for growth. Clinical Nutritional Update 1988;1:6-7.

14 Lily J. Nutritional support in cystic fibrosis. Clinical Nutritio nal Update 1988;1:4-5.

15 Courtney Moore M, Greene HL. Tube feeding of infants and children. Pediatr Clin North Am 1985;32:401-17.

16 Evans J, Cockburn F. Nutritional management of paediatric patients. Baillière's Clinical Gastroenterology 1988;12: patients.

17 Stapleford P. Formula feeding. Paediatric Nursing 1989;6: 14-6.

18 Torrance AD, Harrison C. A controlled study of the performance of five enteral feeding pumps. Fournal of Human Nutrition and Dietetics 1988;1:1-7.

19 Scott C. Home to home: important issues to be resolved. Clinical Nutritional Update 1988;1:8.

$20 \mathrm{McF}$ adzean W. Support for BDA bid for prescribable plastics. Clinical Nutritional Update 1988;1:1.

Cranial computed tomography in tuberculous meningitis

Thirty Californian children with tuberculous meningitis had cranial computed tomography and all showed hydrocephalus, which was said to be moderate or severe in 25 (Waecker and Connor, The Pediatric Infectious Disease fournal 1990;9:539-43). Twenty seven of the children had neurological signs, 10 with impaired consciousness. All were under 6 years old. Previous studies, which have included adults, have shown hydrocephalus to have a $70-100 \%$ sensitivity in the diagnosis of tuberculous meningitis. The specificity is unknown but obviously incomplete. I cannot share the authors' enthusiasm for computed tomography as a primary diagnostic procedure, but it would be useful to do it at some stage in the course of the disease and some children will need a shunt procedure. 